\title{
Análise de procedimentos ambulatoriais realizados em um centro municipal de saúde do Rio de Janeiro
}

\author{
Pollyane R. Miranda, ${ }_{1}^{1 *}$ Mário Rogério S. Santos, ${ }_{1}^{1}$ Ana Cláudia S. Chazan ${ }^{1}$
}

\begin{abstract}
Resumo
A Atenção Primária à Saúde(APS) vem intensificando seu papel na atenção integral e longitudinal. A resolutividade em até $80 \%$ das demandas a qualifica como coordenadora do cuidado. Houve um aumento do modelo Estratégia de Saúde da Família na APS da cidade do Rio de Janeiro de 2009 (7\%) a 2012 (40\%), e neste processo estão inseridos programas de residência em medicina de família e comunidade (MFC), visando à qualificação dos profissionais atuantes na APS. Uma das competências cabíveis ao médico de família e comunidade é a realização de procedimentos e cirurgias ambulatoriais. Foi feita uma análise quantitativa dos procedimentos realizados no Centro Municipal de Saúde Maria Augusta Estrella no período de $1^{\circ}$ de junho a 30 de novembro de 2015, a fim de verificar os mais frequentes, discutindo-se a inserção do residente em MFC neste serviço para formação qualificada. Também foram avaliadas as condições de funcionamento do serviço de cirurgia ambulatorial, ressaltando-se as facilidades e dificuldades. Os procedimentos realizados são compatíveis com os descritos nas principais fontes de orientação de realização de procedimentos/cirurgias ambulatoriais, essenciais à formação do residente. A realização de procedimentos compõe os atributos essenciais à APS, portanto é necessário investimento em estudos posteriores acerca desta competência para aprimorá-la.
\end{abstract}

Descritores: Procedimentos; Cirurgia ambulatorial; Atenção Primária à Saúde; Medicina de família e comunidade; Educação médica.

\begin{abstract}
Analysis of ambulatory procedures performed in a municipal health center from Rio de Janeiro

The Primary Health Care (PHC) has intensified its role in integral and longitudinal attention. The resolution up to $80 \%$ of demands qualifies it as care coordinator. There was an increase in the Family Health Strategy model PHC of the city of Rio de Janeiro from 2009 (7\%) to 2012 (40\%), and the residency programs in family and community medicine (FCM) for the qualification of professionals working in PHC are part of this process. One of the required skills to being a Family and Community Doctor is to be able to conduct procedures and ambulatory surgeries. A quantitative analysis of the procedures performed in the Municipal Health Center Maria Augusta Estrella in the period from June 1st to November 30th, 2015 was made in order to identify the most frequents ones, and also to discuss the inclusion of the resident in FCM at this service to their qualified training. The study has also evaluated the operating conditions of the ambulatory surgery
\end{abstract}

\author{
1. Departamento de Medicina Integral, Familiar e Comunitária. \\ Faculdade de Ciências Médicas. Universidade do Estado do Rio de \\ Janeiro. Rio de Janeiro. RJ, Brasil.
}

\author{
*Endereço para correspondência: \\ Av. Professor Manuel de Abreu, 444, 8 andar \\ Rio de Janeiro, RJ, Brasil. CEP: 20550-170. \\ E-mail: pollyanermiranda@gmail.com
}

Revista HUPE, Rio de Janeiro, 2016;15(3):235-241

doi: 10.12957/rhupe.2016.29449

Recebido em 14/09/2016. Aprovado em 20/12/2016.

service, highlighting the strengths and weaknesses. The procedures performed are consistent with those described in the main guidelines of procedures/ambulatory surgeries, and are essential to the formation of the resident. The performance of such procedures is part of the essential attributes to PHC, hence there is a need to invest in further studies regarding this skill in order to improve it.

Keywords: Procedures; Ambulatory surgery; Primary health care; Family and community medicine; Medical education.

\section{Resumen}

Análisis de los procedimientos ambulatorios realizados en um centro de salud municipal de Rio de Janeiro

La Atención Primaria de Salud (APS) viene intensificando su papel en la atención integral y longitudinal. La resolutividad en hasta el $80 \%$ de las demandas la califica como coordinadora del cuidado. Hubo un aumento del modelo Estrategia de Salud de la Familia en la APS de la ciudad de Río de Janeiro del 2009 (7\%) y 2012 (40\%), y en este proceso se encuentran inseridos programas de residencia en Medicina de Familia y Comunidad (MFC), con interés en la calificación de los profesionales que trabajan en la APS. Una de las competencias del Médico de Familia y Comunidad es la realización de procedimientos y cirugías ambulatoriales. Se hizo un análisis cuantitativo de los procedimientos llevados a cabo en el Centro Municipal de Salud, María Augusta Estrella, en el período del 1 de junio al 30 de noviembre del año 2015, con la finalidad de comprobar los más frecuentes, llevando a discusión, la inserción del residente en MFC en este servicio para su formación calificada. También se evaluaron las condiciones de funcionamiento del servicio de cirugia ambulatoria, destacando las facilidades y dificultades. Los 


\section{Artigo original}

procedimientos realizados son compatibles con los descritos en las principales fuentes de orientación para la realización, de los procedimientos/cirugías ambulatorias y son esenciales para la formación del residente. La realización de los procedimientos forman parte de los atributos esenciales de la APS

\section{Introdução}

A atenção integral às necessidades de saúde de uma população de um território, de forma coordenada e articulada por uma rede de serviços, é um atributo essencial da Atenção Primária à Saúde (APS). ${ }^{1}$

Em 2009, no Município do Rio de Janeiro, a cobertura da ESF era de aproximadamente 7\% de sua população. Iniciou-se uma reforma da APS, representada pela criação das novas clínicas da família e pela implantação das Unidades tipo A (constituídas inteiramente pelo modelo ESF), diferente das unidades tipo B (unidades tradicionais com algumas equipes ESF) e tipo C (unidades tradicionais, sem ESF). ${ }^{2}$

O Centro Municipal de Saúde Maria Augusta Estrella (CMS MAE) é uma unidade do tipo B, cuja área não coberta pela ESF abrange os bairros do Andaraí, Grajaú e parte de Vila Isabel. A área coberta pela ESF abrange a maior parte de Vila Isabel, sendo dividida, até a ocasião deste trabalho, entre quatro equipes: Senador, Conselheiro, Petrocochino e Alto Simão. ${ }^{3}$

A resolutividade em tempo oportuno de até 80\% dessas demandas ${ }^{1}$ por meio da acessibilidade e da continuidade do cuidado qualificado é o que sustenta a APS no lugar de coordenadora do cuidado em saúde, pois o usuário, ao perceber e vivenciar que as intervenções necessárias lhe são oferecidas de forma programada e continuada, cria um vínculo de confiança e adere melhor às recomendações que lhe são feitas.

Às atividades de promoção à saúde e prevenção de doenças e agravos, realizados em âmbito individual, familiar e comunitário, somam-se a execução de procedimentos que eram realizados no hospital, como pequenas cirurgias, atendimentos de urgência, cuidados paliativos e acompanhamento pós-alta hospitalar. ${ }^{1}$

No entanto, um grande volume de procedimentos cabíveis à APS ainda é encaminhado para os níveis secundários e terciários de atenção à saúde. Os processos operacionais de regulação em saúde ainda são incipientes, e requerem aprimoramento e adequação dos encaminhamentos para garantir a qualidade do atendimento aos usuários, assim como a alocação eficiente dos recursos médico-hospitalares. ${ }^{4}$ A construção de uma APS resolutiva contribui para otimizar esse processo.

No que diz respeito à qualificação dos Programas haciéndose necesarias las inversiones en estudios posteriores a cerca de esta competencia para mejorarla.

Palabras clave: Procedimientos; Cirugía ambulatoria; Atención primaria de la Salud; Medicina de familia y comunidad; Educación médica.

de Residência em Medicina de Família e Comunidade (PRMFC), a Sociedade Brasileira de Medicina de Família e Comunidade (SBMFC) desenvolveu, com base em experiências nacionais e internacionais, diretrizes para orientar a formação em Medicina de Família e Comunidade (MFC) - o Currículo Baseado em Competências. ${ }^{5}$

No campo da Atenção à Saúde, a realização de procedimentos ambulatoriais representa uma das áreas de competência do grupo de Atenção Individual. Nessa competência, por exemplo, a drenagem de abscesso é classificada como essencial, enquanto o agulhamento de pontos de gatilho, como avançada. 5

O Município do Rio de Janeiro possui uma Carteira de Serviços voltada aos profissionais, aos gestores e à população que visa nortear as ações de saúde na APS oferecidas à população da cidade. Dentre as descrições dos serviços prestados, está discriminada a seção de Procedimentos e Cirurgias Ambulatoriais. ${ }^{6}$

Os procedimentos clínicos e cirúrgicos realizados nas Unidades Básicas de Saúde (UBS) estão relacionados aos agravos comumente atendidos na APS. Para tais procedimentos, há uma listagem de equipamentos, materiais, medicamentos e insumos necessários nas UBS. Além disso, a realização deles deve ocorrer com base em protocolos e evidências científicas.

O objetivo deste artigo é apresentar uma análise das pequenas cirurgias e procedimentos realizados no CMS MAE, campo de prática para a formação em MFC, à luz das competências que este médico deve desenvolver e da carteira de serviços do Município do Rio de Janeiro.

\section{Métodos}

Realizou-se uma análise quantitativa dos procedimentos e cirurgias ambulatoriais realizados no CMS MAE no período de $1^{\circ}$ de junho a 30 de novembro de 2015.

Os dados foram obtidos da Planilha de Procedimentos do CMS MAE ${ }^{8}$ e do Boletim de Produção Ambulatorial (BPA) da ESF do CMS MAE. ${ }^{9}$

A busca foi feita por sexo, idade, condição de saúde relacionada ao procedimento, origem do paciente(ESF, Modelo Tradicional, outro), e se houve ou não envio do material para análise histopatológica. 
As faixas etárias foram divididas de acordo com o ciclo de vida: de 0 a 12 anos (crianças), 13 a 18 anos (adolescentes), 19 a 45 anos (adultos jovens), 46 a 59 anos (adultos de meia idade), 60 anos ou mais (idosos).

Para classificação dos tipos de procedimentos e cirurgias ambulatoriais, foram utilizados a Carteira de Serviços da SMS-RJ, ${ }^{5}$ o Caderno de Atenção Primária número 30 - Procedimentos, ${ }^{6}$ e o Currículo Baseado em Competências para Medicina de Família e Comunida- de. ${ }^{5}$ A tabela 1 mostra a classificação dos procedimentos de acordo com essas três fontes.

Também foram excluídos do total alguns procedimentos predominantemente clínicos atendidos no dia a dia das equipes, como as coletas de material para exame citopatológico (papanicolaou), por não mobilizarem especificamente o serviço de pequenos procedimentos do CMS MAE.

Tabela 1. Classificação de procedimentos e cirurgias ambulatoriais de acordo com o currículo baseado em competências da SBMFC, com o Caderno de Atenção Primária no 30 e com a Carteira de Serviços da APS da SMS-RJ*

\begin{tabular}{|c|c|c|c|c|}
\hline $\begin{array}{l}\text { Lista de procedimentos e } \\
\text { cirurgias ambulatoriais }\end{array}$ & $\begin{array}{l}\text { Competências essenciais } \\
\text { do currículo baseado em } \\
\text { competências da SBMFC }\end{array}$ & $\begin{array}{l}\text { Competências desejáveis } \\
\text { do currículo baseado em } \\
\text { competências da SBMFC }\end{array}$ & $\begin{array}{c}\text { Caderno de } \\
\text { Atenção Primária } \\
\text { no } 30\end{array}$ & $\begin{array}{c}\text { Carteira de } \\
\text { Serviços da APS - } \\
\text { SMS-RJ }\end{array}$ \\
\hline Drenagem de abscesso & $x$ & & $x$ & $x$ \\
\hline $\begin{array}{l}\text { Exérese de pequenas lesões } \\
\text { superficiais }\end{array}$ & $x$ & & $x$ & $x$ \\
\hline Cantoplastia & $x$ & & $x$ & $x$ \\
\hline $\begin{array}{l}\text { Cauterização química de } \\
\text { verruga viral }\end{array}$ & $x$ & & & $x$ \\
\hline $\begin{array}{l}\text { Anestesia local e bloqueios } \\
\text { anestésicos de quirodáctilos } \\
\text { e pododáctilos }\end{array}$ & $x$ & & & \\
\hline Remoção de cerume & $x$ & & $x$ & $x$ \\
\hline Retirada de corpo estranho & $x$ & & $\mathrm{x}$ & $x$ \\
\hline Frenectomia & $x$ & & & \\
\hline Tamponamento nasal anterior & $x$ & & & $x$ \\
\hline $\begin{array}{l}\text { Procedimentos de urgência } \\
\text { (ex: compressões e } \\
\text { imobilizações) }\end{array}$ & $x$ & & $x$ & \\
\hline $\begin{array}{l}\text { Inserção de dispositivo } \\
\text { intrauterino }\end{array}$ & $x$ & & & $x$ \\
\hline Biópsias de pele & & $x$ & $x$ & $x$ \\
\hline Tratamento de calo plantar & & $x$ & & $x$ \\
\hline $\begin{array}{l}\text { Remoção de molusco } \\
\text { contagioso }\end{array}$ & & & $x$ & \\
\hline Aspiração de cisto sinovial & & $x$ & & \\
\hline Exérese de pólipo uterino & & & & $x$ \\
\hline Infiltração intra-articular & & $\mathrm{x}$ & & \\
\hline $\begin{array}{l}\text { Cateterismo vesical, passagem } \\
\text { de sonda nasogástrica }\end{array}$ & & $x$ & $x$ & $x$ \\
\hline $\begin{array}{l}\text { Tamponamento nasal } \\
\text { posterior }\end{array}$ & & $\mathrm{x}$ & & \\
\hline
\end{tabular}

*Adaptado das fontes citadas. 


\section{Artigo original}

\section{Resultados}

A coordenação do serviço de cirurgia ambulatorial do CMS MAE é feita por um dos preceptores da Residência em MFC-UERJ, e os procedimentos são realizados em um turno de quatro horas, semanalmente, em uma sala que possui 5,69 $\mathrm{m}^{2}$ (2,14 $\mathrm{m}$ x 2,66 m), que dispõe de uma maca, uma pia; um foco clínico; uma mesa de Mayo; um ar condicionado, uma lixeira e um dispositivo apropriado de descarte para objetos perfurocortantes.

Os materiais descartáveis são acondicionados em um gaveteiro móvel e as bandejas de materiais cirúrgicos ficam no centro de esterilização. Ambos são transportados pelos profissionais até a sala no turno destinado aos pequenos procedimentos.

Durante o período de $1^{\circ}$ de junho a 30 de novembro de 2015, 165 procedimentos foram realizados pelo serviço, sendo 93 em mulheres (56,36\%) e 72 em homens (43,64\%).

Observando-se a divisão por faixa etária, a maioria dos pacientes atendidos possuía 60 anos de idade ou mais, independente do sexo (37,5\% homens e 32,26\% mulheres), constituindo $34,55 \%$ do total. Apenas $12,12 \%$ dos procedimentos foram feitos em crianças ou adolescentes, todavia todas as idades mostraram representatividade (Tabela 2).

Além disso, por meio das informações do BPA e da planilha de serviço utilizada pela equipe do CMS MAE, os pacientes puderam ser estratificados de acordo com sua origem de encaminhamento para o procedimento. Do total de 165 pacientes, $115(69,7 \%)$ pertenciam à ESF, 36 (21,82\%) ao Modelo Tradicional e $14(8,48 \%)$ a outros serviços externos ao CMS MAE (denominados "Fora de Área").

Na tabela 3 podemos observar a distribuição dos usuários de acordo com a origem da referência. Observa-se que a Equipe Petrocochino foi a responsável pela maior demanda pelo serviço, constituindo $30,43 \%$ dos pacientes procedentes da ESF e 21,21\% do total de pacientes atendidos.

Em relação à classificação dos tipos de procedimentos, observou-se que a maior busca foi por realização de exérese de lesões superficiais como cisto, lipoma, nevo, dermatose papulosa nigra, fibroma mole, verruga, millium, entre outras. Essa categoria correspondeu a $47,27 \%$ do total de procedimentos realizados; sendo que lesões enviadas para biópsia foram contabilizadas separadamente, constituindo $12,12 \%$ do total (Tabela 4).

\section{Discussão}

A descrição dos procedimentos e cirurgias ambulatoriais da Carteira de Serviços prestados à APS do Município do Rio de Janeiro, ${ }^{6}$ do Caderno de Atenção Primária - Procedimentos, ${ }^{7}$ e do Currículo Baseado em Competências para Medicina de Família e Comu-

Tabela 2. Pacientes submetidos aos procedimentos segundo sexo e idade (\%) (18,9 $^{-1}$

\begin{tabular}{|c|c|c|c|}
\hline Idade & Homens (\%) & Mulheres (\%) & Total (\%) \\
\hline $\mathbf{0}$ a 12 anos & $4(5,56)$ & $4(4,30)$ & $8(4,85)$ \\
\hline 13 a 18 anos & $5(6,94)$ & $7(7,53)$ & $12(7,27)$ \\
\hline 19 a 45 anos & $17(23,61)$ & $24(25,80)$ & $41(24,85)$ \\
\hline 46 a 59 anos & $19(26,39)$ & $28(30,11)$ & $47(28,48)$ \\
\hline$\geq 60$ anos & $27(37,50)$ & $30(32,26)$ & $57(34,55)$ \\
\hline Total & $\mathbf{7 2 ( 1 0 0 )}$ & $\mathbf{9 3 ( 1 0 0 )}$ & $165(100)$ \\
\hline
\end{tabular}

*Porcentagem de pacientes entre parênteses.

Tabela 3. Pacientes submetidos aos procedimentos segundo origem da referência (\%)*8,9

\begin{tabular}{|c|c|c|c|c|c|c|}
\hline \multicolumn{4}{|c|}{ Equipes da ESF } & \multirow{2}{*}{$\begin{array}{l}\text { Modelo } \\
\text { tradicional }\end{array}$} & \multirow{2}{*}{ Fora de Área } & \multirow{2}{*}{ Total } \\
\hline Senador & Conselheiro & Petrocochino & Alto Simão & & & \\
\hline $33(20,00)$ & $21(12,73)$ & $35(21,21)$ & $26(15,76)$ & \multirow{2}{*}{$36(21,82)$} & \multirow{2}{*}{$14(8,48)$} & \multirow{2}{*}{$165(100)$} \\
\hline \multicolumn{4}{|c|}{ Total ESF $115(69,7)$} & & & \\
\hline
\end{tabular}

*Porcentagem de pacientes entre parênteses. 
nidade $^{5}$ são distintas, mas apresentam alguns pontos em comum.

Os procedimentos realizados no serviço do CMS MAE estão distribuídos nas três fontes, não sendo possível utilizar apenas uma delas como referência. Ressalta-se que a Carteira de Serviços do Rio de Janeiro traz a descrição de uma listagem abrangendo também procedimentos técnicos e administrativos da Unidade de Saúde como um todo, que fogem ao escopo deste trabalho e por isso não foram contabilizadas.

A análise dos procedimentos efetuados no CMS MAE revelou que a maioria da demanda é adulta ou idosa, indo ao encontro da literatura que revela que o processo de envelhecimento populacional agrega mais desafios aos serviços de saúde - como a frequente apresentação de pessoas com multimorbidades. ${ }^{2}$ Somado ao fato de que todas as faixas etárias foram representadas no estudo, reforça-se o "olhar para família" no papel coordenador, longitudinal e integral da APS responsável por uma população adscrita. ${ }^{1}$

Em todas as faixas etárias o número de mulheres é igual ou superior ao de homens, refletindo a vivência de uma maior busca habitual dos serviços de saúde no âmbito da APS pelas mulheres quando comparado ao volume da demanda pelos homens. ${ }^{2,10}$ Esse comportamento já foi discutido e analisado em outros estudos e envolve diversos aspectos relacionados ao universo masculino e ao cotidiano dessa população. ${ }^{10}$ Além disso, destaca-se o incipiente foco à saúde do homem nos serviços da APS - tradicionalmente mais voltada a destacar o programa de saúde da mulher., ${ }^{2,10}$

Ainda que disponível para todos os usuários do CMS MAE (ESF e Modelo Tradicional), o serviço de pequenos procedimentos foi mais usufruído pelos pacientes da ESF, apesar de a população pertencente ao Modelo Tradicional ser maior. Tal resultado pode refletir diferença na indicação dos procedimentos, na acessibilidade ao serviço, bem como na relação de vínculo e confiança dos usuários aos médicos que indicam os procedimentos.

Dentre as equipes da ESF, a maior demanda pelo serviço de procedimentos foi a da Equipe Petrocochino, que também é a equipe com o maior número de pacientes cadastrados; sendo o resultado, portanto, considerado esperado.

Quanto aos usuários fora da área do CMS MAE, estes possuíam alguma ligação com o PRMFC - como ser adscrito a outra UBS campo de prática para Residência Médica que não possui estrutura para realização de cirurgia ambulatorial, por exemplo.

Em relação aos diferentes tipos de procedimentos que foram realizados, percebe-se a expressividade da demanda por retirada e biópsia de pequenas lesões superficiais de pele (nevos, lipomas, cistos sebáceos, lesões suspeitas de malignidade, etc.) seguida por drenagem de abscesso, retirada de cerume, tratamento de calo plantar e cauterização química (Tabela 4). Isso demonstra a necessidade do conhecimento de técnica cirúrgica e

Tabela 4. Tipos de procedimentos realizados e proporção quantitativa (\%)*8,9

\begin{tabular}{|l|c|}
\hline Exérese de pequenas lesões superficiais ${ }^{1}$ & $78(47,27)$ \\
\hline Biópsias de pele & $20(12,12)$ \\
\hline Drenagem de abscesso & $15(9,09)$ \\
\hline Retirada de cerume & $12(7,27)$ \\
\hline Tratamento de calo plantar ${ }^{2}$ & $9(5,45)$ \\
\hline Cauterização química & \\
\hline Inserção de Dispositivo Intrauterino & $9(5,45)$ \\
\hline Cantoplastia & $8(4,85)$ \\
\hline Remoção de molusco contagioso & $5(3,03)$ \\
\hline Aspiração de cisto sinovial & $3(1,82)$ \\
\hline Exérese de pólipo uterino & $3(1,82)$ \\
\hline Infiltração intra-articular & $2(1,21)$ \\
\hline TOTAL & $1(0,61)$ \\
\hline *Porcentagem de pacientes entre parênteses & $165(100)$ \\
\hline
\end{tabular}




\section{Artigo original}

de uma boa formação na graduação para o residente em MFC. ${ }^{5}$ Pela maior frequência desses atos, é viável para o residente adquirir habilidade prática e corrigir algumas deficiências elementares. Assim, poderá atuar de forma apropriada sobre essas condições de saúde como médico de família e comunidade.

Todas as três fontes utilizadas para a descrição dos tipos de procedimentos mencionam procedimentos de urgência como, por exemplo: drenagem de abscessos, suturas e tratamento de feridas superficiais, como essenciais para uma assistência eficiente prestada em MFC. Porém, devido à sua natureza de atendimento imediato, esses tipos de procedimentos não foram contabilizados pela planilha do Google Drive do serviço do CMS MAE, ${ }^{8}$ por não serem agendados.

Além disso, perceberam-se poucos registros em prontuário desses procedimentos de urgência, o que pode ser explicado pelo registro indevido ou não registro dos procedimentos realizados. Assim, é provável que tenha ocorrido subestimativa do número real em alguns dos quantitativos.

Outro ponto a ser destacado nessa contagem dos procedimentos é que não houve acesso ao BPA do Modelo Tradicional por questões de processo administrativo (não digitalizado), tendo ocorrido possivelmente subnotificação dos procedimentos realizados por alguns dos médicos do Modelo Tradicional em relação à inserção de dispositivo intrauterino (DIU), exérese de pólipo uterino e cauterização química. Os demais procedimentos listados na tabela 4 são feitos apenas aos pacientes da ESF ou àqueles encaminhados ao serviço de procedimentos/cirurgias ambulatoriais.

A criação de uma Carteira de Serviços ${ }^{6}$ pelo Município do Rio de Janeiro tem servido como um norte para alcance de um atendimento mais integral e resolutivo à população, à medida que todas as ações e serviços descritos nesta carteira sejam ofertados com qualidade, uma vez que estes atributos são essenciais à APS. ${ }^{2}$

A realização de procedimentos ambulatoriais contribui significativamente para afirmação desses atributos, evitando o estrangulamento dos serviços dos outros níveis de atenção.?

O serviço de pequenos procedimentos do CMS MAE realiza a maioria dos procedimentos descritos, cooperando para formação adequada de profissionais especializados e capacitados para atuar na APS, por meio do Programa de Residência em MFC.

Em relação ao serviço de pequenos procedimentos do CMS MAE, observam-se algumas facilidades e desafios. Entre as facilidades existe a disponibilidade de pro- fissionais capacitados sob a orientação de preceptores do PRMFC-UERJ para a realização dos atendimentos, havendo oportunidade do próprio médico residente da equipe do paciente realizar o ato, fortalecendo o vínculo e garantindo a execução plena dos atributos essenciais à APS.11 Há também o acesso ao material necessário no CMS MAE para execução dos procedimentos, favorecendo o funcionamento do serviço, assim como é disponbilizado um contato externo para encaminhamento das biópsias realizadas.

Entre os desafios enfrentados pelo serviço, citamos uma estrutura física inadequada e não exclusiva para a realização de pequenos procedimentos, o que acarreta ocupação da sala por outros serviços do CMS MAE. Tudo isso gera a impossibilidade da guarda do material no local, falta de espaço para os profissionais em formação acompanharem os procedimentos e realizarem atendimentos. Além disso, há a falta de alguns materiais acessórios para otimização dos procedimentos e alcance potencial de melhores resultados. Todos esses problemas levam ao desgaste físico e mental dos profissionais envolvidos e prejudicam a qualidade do serviço prestado.

\section{Conclusão}

Os procedimentos realizados no CMS MAE contemplam aqueles descritos na Carteira de Serviços prestados à APS do Município do Rio de Janeiro, ${ }^{6}$ no Caderno de Atenção Primária - Procedimentos, ${ }^{7}$ e no Currículo Baseado em Competências para Medicina de Família e Comunidade. ${ }^{5}$ Assim, realiza-se o serviço proposto à responsabilidade da APS no sentido de evitar encaminhamentos desnecessários à Atenção Especializada.

A oferta de procedimentos na APS contribui para a integralidade do cuidado aos pacientes. Além disso, a APS possui o atributo da longitudinalidade como trunfo para melhor acompanhamento pré e pós-procedimento, atingindo-se provavelmente melhores resultados do que em serviços de atendimentos pontuais, frutos de encaminhamentos.

A demanda pela realização de procedimentos ambulatoriais em várias situações diferentes (idade, gênero, história clínica e pessoal, etc.) indica que o médico de família e comunidade deve saber verificar a indicação ou não do ato, além de exigir o domínio da habilidade para realização plena dos procedimentos mais frequentes cabíveis à APS. A realização sucessiva dos procedimentos mais demandados permite o médico residente adquirir boa prática para execução dos mesmos e o capacita a realizá-los após o término de sua pós-graduação. 
Apesar de contar com profissionais capacitados e dispor do material básico para execução dos procedimentos, o serviço de procedimentos e cirurgias ambulatoriais do CMS MAE enfrenta uma série de desafios ao seu funcionamento pleno. A estrutura física e o funcionamento de uma unidade mista (Unidade B) representam pontos cruciais de empecilho à melhor organização do setor, uma vez que o Modelo Tradicional e a ESF possuem organizações distintas dos seus processos de trabalho.

Por fim, ressalta-se que este trabalho traz uma análise focal e inicial da perspectiva de um campo de atuação importante do Médico de Família e Comunidade. Uma limitação encontrada foi a ausência de estudos publicados na área, para efeito de correlação, por exemplo.É crucial que haja incentivo para estudos e publicações sobre procedimentos no âmbito da APS a fim de enriquecer a literatura deste tema.

\section{Agradecimentos}

Agradeço às minhas amigas e colegas Residentes Camila Maria da Silva Oliveira e Caroline Fagundes Campana, que participaram do apoio à construção deste estudo durante o processo de trabalho do CMS MAE.

Agradeço aos meus orientadores Mário Rogério da Silva Santos e Ana Cláudia Santos Chazan, que muito contribuíram na elaboração das ideias deste trabalho.

Agradeço ao meu preceptor Philipp Rosa de Oliveira, responsável direto por minha experiência em cirurgia e procedimentos ambulatoriais durante o segundo ano de Residência em MFC, o que foi fundamental para minha construção como Médica de Família e Comunidade.

\section{Referências}

1. Almeida PF, Fausto MCR, Giovanella L. Fortalecimento da atenção primária à saúde: estratégia para potencializar a coordenação dos cuidados. Rev. Panam Salud. 2011:29(2)84-85

2. Harzeim E; Lima KM, Hauser L. Reforma da Atenção Primária a Saúde no Rio de janeiro- Avaliação dos três primeiros anos de clínica da Família. Rio Grande do Sul; 2013.

3. Secretaria Municipal de Saúde e Defesa Civil (SMSDC) - Coordenadoria Geral de Saúde da Área Programática 2.2. CAP 2.2. Disponível em: <http://cap22tijuca.blogspot.com.br/p/ unidades.html>. Acesso em: 02 dez. 2015.

4. Vilarins GCM, Shimizu HE, Gutierrez UM. A regulação em saúde: Aspectos conceituais e operacionais. Saúde em Debate. 2012;36(95):640-647.

5. Sociedade Brasileira de Medicina de Família e Comunidade. Currículo Baseado em Competências para Medicina de Família e Comunidade; 2015.

6. Secretaria Municipal de Saúde e Defesa Civil (SMSDC) Superintendência de atenção Primária. Carteira de Serviços prestados na Atenção Primária; $1^{\text {a }}$ edição; 2011.

7. Brasil. Caderno de atenção Primária - Procedimentos. Brasília, DF: Ministério da Saúde; 2011.

8. Secretaria Municipal de Saúde e Defesa Civil (SMSDC) - Coordenadoria Geral de Saúde da Área Programática 2.2. Centro Municipal de Saúde Maria Augusta Estrella. Disponível em: $<$ http://smsdc-cmsmaestrella.blogspot.com.br>. Acesso em:02 dez. 2015.

8. Centro Municipal de Saúde Maria Augusta Estrella. Planilha Procedimentos - CMS MAE Disponível em: <https://docs. google.com/spreadsheets/d/1anVKV30nMsPZxWhY5ZHqs9wQTSjXeBoRJQU6sUVbfO0/edit\#gid=0 >. Acesso em: 01 dez. 2015.

9. Centro Municipal de Saúde Maria Augusta Estrella. Boletim de Produção Ambulatorial (01/06/2015 a 30/11/2015). Disponível em: VITA his CARE E-Health Solution. Acesso em: 02 dez. 2015.

10. Alves RF, Silva RP, Ernesto MV, et al. Gênero e saúde: o cuidar do homem em debate. Psicologia: Teoria e Prática. 2011;13(3):152-166.

11. Jaakkimainen RL, Sood PR, Schultz SE. Office-based procedures among urban and rural family physicians in Ontario. Can Fam Physician. 2012;58(10):578-587 